\title{
Modeling, Design and Simulation of Micro-Force Sensor Based on PVDF
}

\author{
Yiyang Liu, ${ }^{1, a}$, Liu Liü, ${ }^{2, b}$ Zengcai $X u^{2, c}$, Xinrui $S^{2, d}$ \\ ${ }^{1}$ Shenyang Institute of Automation, Chinese Academy of Sciences, \\ Nanta St.114, Shenyang 110016, P. R. China \\ ${ }^{2}$ Northeastern University, \\ Wenhua Road 3-11, Shenyang 110819 P. R. China \\ asialiuyiyang@sia.cn, bliuliu@sia.cn, ${ }^{c} 18842409017 @ 163 . c o m,{ }^{d}$ sxrjordon23@163.com
}

\begin{abstract}
Keywords: Micro force sensor; Micro assembly; Micromanipulation
Abstract. At present, by existing sensors, sub- $\mu \mathrm{N}$ micro-force in micro-assembly is not able to be reliably measured. In this paper, using PVDF film as the sensitive element and using micro-force sensor based on PVDF as the research object are to establish the model of PVDF sensor and analyze the relationship between output charge of PVDF and the force acting on it. Then to design the corresponding signal processing circuit, then simulate the circuit. Simulation results show design of the signal processing circuit is effective and modeling of PVDF sensor is correct. Thus, the paper provides a feasible solution in micro-force sensing and feedback control for micro-assembly and micro-manipulation.
\end{abstract}

\section{Introduction}

Micro Electric Mechanical System (MEMS) is a cutting edge technology in twenty-first century. Now it is developing to become a new research area including a set of micro mechanical, micro sensors, micro controllers, micro actuators, signal processing and intelligent control. Because its work can be carried out within the narrow space and not disrupt the working environment and the objects, the application will be involved in the aerospace, military, biomedical engineering, space exploration, the deep sea exploration and many other areas. The mature technology and industrialization will have a profound impact on the national economic construction, national defense construction and social development.

But device in the process of assembling MEMS cheaply has not yet been developed, because the structure of micro measurement is brittle. For example, Micro lens of MEMS optical switch components used in WMD optical network system is fragile and easily broken [1][2]. As shown in Figure 1, Micro mirrors are very important components in Micro-Opto Electro Mechanical System (MOEMS). Their size is tiny (the typical size is $100 \mu \mathrm{m} \times 200 \mu \mathrm{m}$ ), and mirror Orientation and control structure can be monolithically integrated. The micro mirror is not only very important for MEMS system, but also plays a huge role in daily production and life. But it can be broken in reaction of micro Newton.. Because there is no reliable three-dimensional micro contact force sensing method in resolution of micro Newton $(s u b-\mu N)$, it is low efficiency and high breakage rate to assemble micro lens, and difficult to achieve automated micro assembly.

Moreover, in biomechanics research in the field of biomedical, single-celled operation [3][4][5], and micro manipulation in the field of aerospace[6][7] it is also a difficult problem to measure micro force in micro environment. Therefore, this paper is based on PVDF material to establish a theory model about micro force $\mathrm{F}$ acted on PVDF micro force sensor and electric charge Q produced by PVDF. 


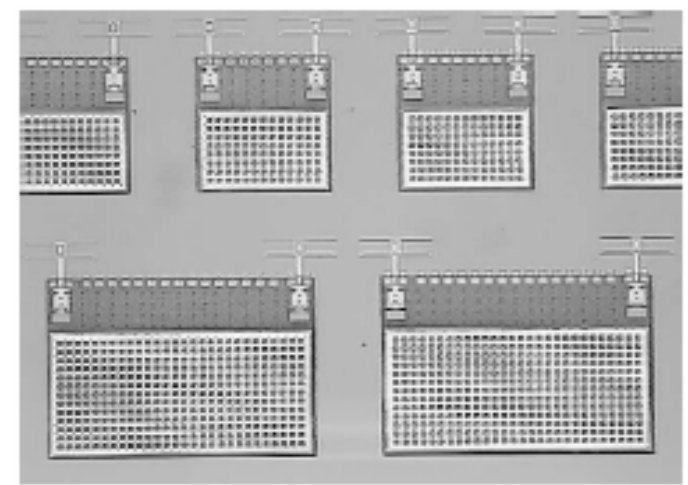

Fig.1. Micro mirror array.

\section{PVDF BASIC CHARACTERISTICS AND MODELING ANALYSIS}

Since PVDF (Polyvinylidene Fluoride) was discovered by the Japanese scholar Kawai in 1969, it attracted the great interest of scholars, and was widely carried on the thorough research in the preparation process, properties and practical application in virtue of its characteristics of wide frequency response, good dynamic range, high output voltage, good stability, impact resistance, and not easy to aging etc[8]. These characteristics also make PVDF have the incomparable superiority on the design of micro force sensor compared with other piezoelectric materials.

Structure diagram of PVDF micro-force sensor is shown in Figure 1. In the figure, $\mathrm{W}$ is PVDF width. $L$ is PVDF length. $h$ is PVDF Thickness. $A(A=L \times W)$ is surface area of PVDF. $a(a=W \times h)$ is cross section area of PVDF. L0 is length of probe. $\mathrm{d} 31$ is piezoelectric constant of PVDF.

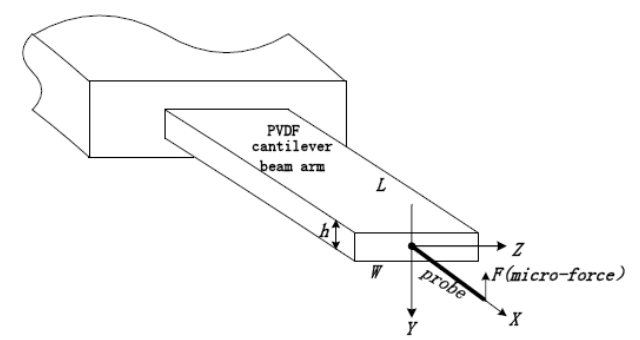

Fig.2. Schematic diagram of a PVDF sensor's structure

According to the piezoelectric effect, charge Q on the surface of PVDF is

$$
Q=\int d_{31} \delta d A
$$

(1)

$$
\delta=\frac{2}{W h} \int_{0}^{\frac{h}{2}} \delta_{1} d a
$$

Tension force in any point of PVDF film is

$\delta_{1}=\frac{M}{I_{z}}$

Inertia moment of PVDF film is

$$
I_{z}=\frac{W h^{3}}{12}
$$

Bending moment of PVDF film is

$M=F\left(L-x+L_{0}\right)$

According to the above formulas, formula (1) can be written as

$Q=\frac{3 d_{31} L\left(L+2 L_{0}\right)}{2 h^{3}} F$

Because the parameters of the formula (6) are known, charge Q is linear with force F. 


\section{DESIGN OF SIGNAL PROCESSING CIRCUIT}

When the PVDF film sensor is deformed or subjected to stress, the amount of charge generated in the polarization direction is rather little, only tens or hundreds of pc (about pico-charge) basically. Therefore, the role of first level amplifying circuit of front-end circuit is crucial. It will not only make the charge signal into voltage signal, but also amplify the original charge signal. The original charge signal only passes a charge amplifying circuit to amplify and convert in order to further enlarge, de-noise, transfer or gather. Therefore, secondary circuit of front-end circuit is designed as a voltage amplifying circuit to amplify the first level signal. Meantime, because the most serious frequency of interference signal is $50 \mathrm{~Hz}$ in this design, the third level is a $50 \mathrm{~Hz}$ trap circuit.

However, the useful signal is amplified through the front-end circuit, the noise is amplified too. A low-pass circuit is chose to filter high frequency noise. Besides, the rear circuit is to amplify the useful signal again in order to be convenient for data collection and computer to process signal. To sum up, the schematic diagram of signal processing circuit is shown in figure 3 .

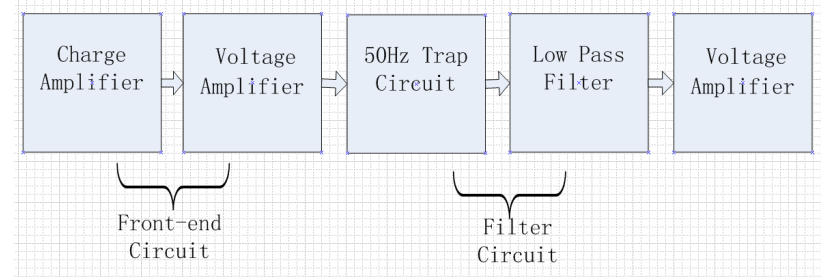

Fig. 3. Schematic diagram of the signal processing circuit

When the PVDF film sensor becomes deformed, the charge will appear on the surface of the electrode. And the amount of charge gathered on the surface of two poles are equal and they have the opposite polarity. Therefore, PVDF thin film can be regarded as a static charge generator with capacitance properties. The equivalent circuit[9] is shown in Figure 4. Ca is the equivalent capacitance for the PVDF thin films, and Ra is the equivalent resistance for the PVDF thin films.

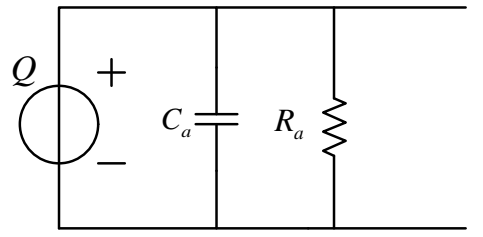

Fig. 4. The equivalent circuit of PVDF piezoelectric thin film

Considering actual situation and equivalent circuit of PVDF film, equivalent circuit of charge amplifier[10] can be shown in Figure 5.

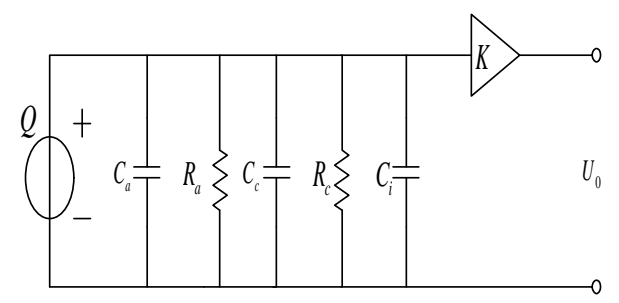

Fig. 5. The equivalent circuit of charge amplifier

Where $\mathrm{Cc}$ and $\mathrm{Rc}$ are the distributed capacitance and insulation resistance which connects the cable. $\mathrm{Ci}$ is the input capacitance of the operational amplifier.

Because the direct charge or voltage signal output from PVDF piezoelectric thin film is very weak, only when the PVDF film sensor and the matching front-end circuit are connected, the signal output will be complete. And at the same time, because the internal resistance of PVDF piezoelectric thin films

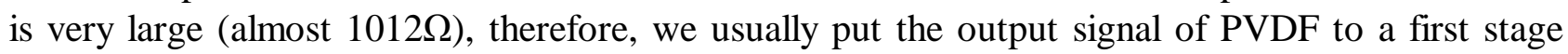
amplifying circuit to transform it into a lower impedance output signal, and then send it to the following amplifying circuit. 
The key part of front-end circuit of PVDF sensor is the first level amplifying circuit. The main functions of it are as follows.

(a) By matching with impedance of PVDF sensor, it can exchange the high impedance input into low impedance output.

(b) Transform the weak charge into voltage signal and amplify the voltage signal.

In order to satisfy the above two points, we choose AD544 to complete the design of charge amplifier. According to its working principle, the actual circuit of the charge amplifier uses negative feedback. Considering the size of the measured physical quantity, and ensure that the post amplifier will not be saturation as the input signal is too large. We choose the $100 \mathrm{pF}-10 \mathrm{nF}$ capacitor to provide negative feedback. Because the capacitor negative feedback circuit is equivalent to open in DC work and is sensitive to the cable noise, the amplifier zero drift is relatively large. $\mathrm{n}$ order to improve the working stability of the amplifier, we can connect parallel a large number of resistances at both ends of the feedback capacitor (usually be 108-1012 $\Omega$ ) to provide direct feedback. So the equivalent charge amplifier circuit with feedback can be shown in Figure 6.

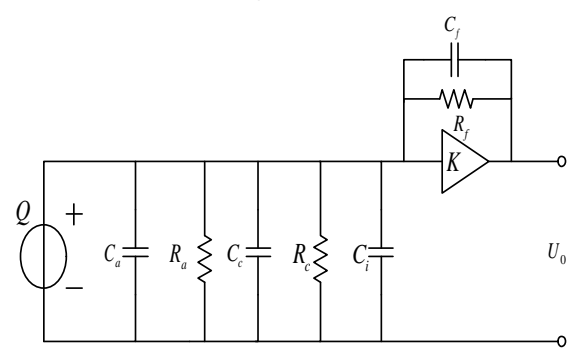

Fig.6. The equivalent charge amplifier circuit with feedback

Considering that there is no charge source directly in the simulation software of Multisim and the characteristics of PVDF, we use pulse current source to replace the charge source signal in the simulation [11].

The equivalent capacitance of PVDF thin films used in this paper is about $100 \mathrm{pF}$, and the equivalent

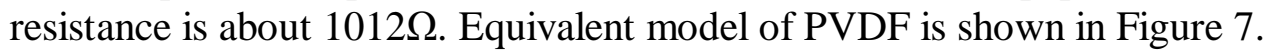

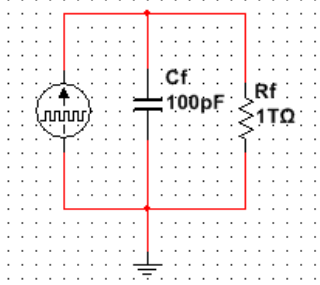

Fig.7. Equivalent model of PVDF

Based on the equivalent model of PVDF, the simulation circuit and the results of the charge amplifier are shown in Figure 8 and Figure 9.

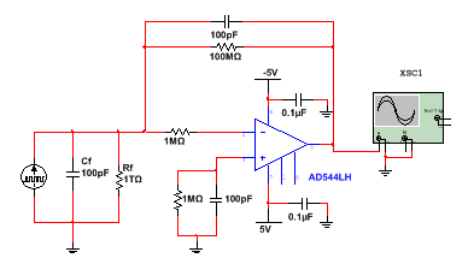

Fig.8. Simulation circuit of the charge amplifier 


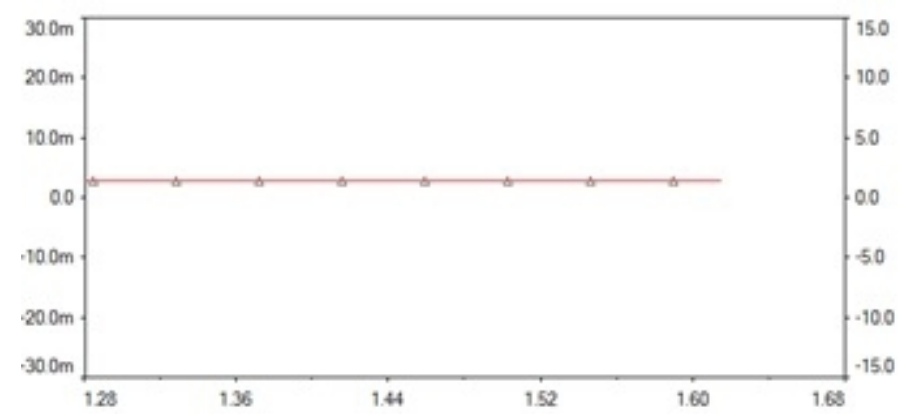

Fig.9. Simulation results of the charge amplifier

In Figure 8, 9, when the current source input is very small, pulse time will be short, according to the formula:

$$
Q=\int_{0}^{t} i(t) d t=I t
$$

In the above formula, I is the pulse output. That is PVDF generates little charge, which can achieve sub pc level. So the force applied on the PVDF capacity is small which can reach sub- $\mu \mathrm{N}$ level. Through the charge amplifier, PVDF film completes the impedance matching, and we get the amplified output.

Considering the most serious $50 \mathrm{~Hz}$ noise in real test, we design a double $\mathrm{T}$ trap circuit to filter $50 \mathrm{~Hz}$ noise. That is shown in Figure 10.

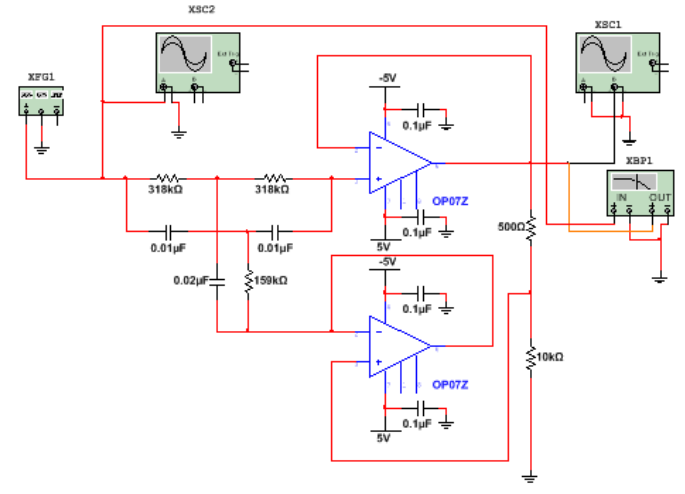

Fig.10. $50 \mathrm{~Hz}$ trap circuit

By setting the function generator, we select the $50 \mathrm{~Hz}$ sine wave as the input. The output on an oscilloscope and oscillographic characteristics instrument are shown in Figure 11 and Figure 12.

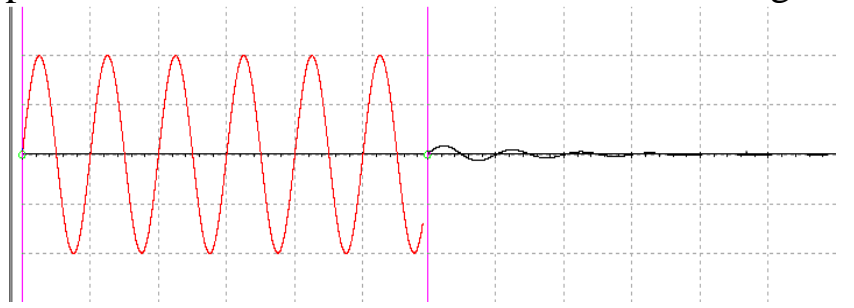

Fig.11. The output on an oscilloscope

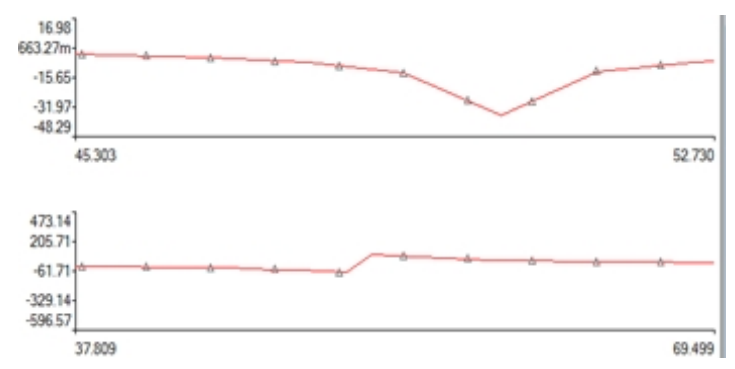

Fig.12. Potter tester output 
In Figure 11, the red line on the left side represents the $50 \mathrm{~Hz}$ input and the black line on the right side represents filter output. We can see from Figure 11 and Figure 12 that after using a trap circuit, test signal through the trap circuit completely removes the $50 \mathrm{~Hz}$ interference.

Through the front-end circuit the useful signal is amplified, and the noise is also amplified. Because the PVDF sensor needs be used in static, quasi-static or low frequency circumstance, a low pass circuit is designed to filter the high frequency noise[12]. Simulation schematic and the simulation results are shown in Figure 13 and Figure 14.

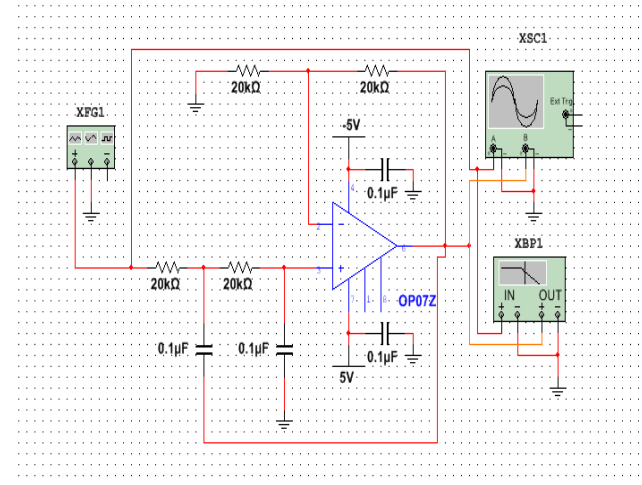

Fig.13. Simulation schematic of low pass circuit

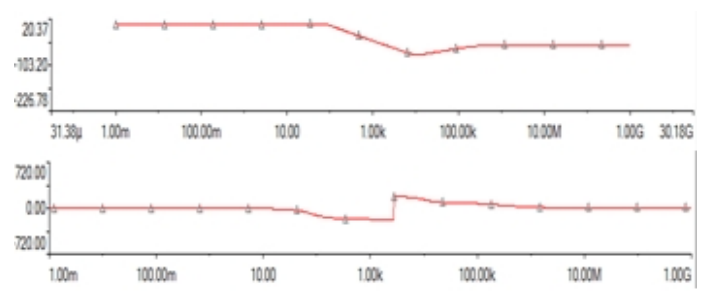

Fig.14. The simulation results of low pass circuit

\section{Summary}

From the above analysis of PVDF modeling and signal processing circuit, we can get the following conclusions.

(a) Through the analysis and modeling of PVDF thin film, the relationship between the force F and the generated charge $\mathrm{Q}$ is proportional linear.

(b) It is reasonable to use pulse current source to replace charge source equivalently, because the results of the theoretical analysis and simulation are the same.

(c) $50 \mathrm{~Hz}$ trap circuit can filter the frequency of $50 \mathrm{~Hz}$ interference effectively.

(d) Low pass circuit can make the signal under $101 \mathrm{~Hz}$ pass, so as to effectively filter the high frequency noise.

In conclusion, signal processing circuit we design can reliably measure the micro force. In this paper, using PVDF film as the sensitive element and using micro-force sensor based on PVDF as the research object are to establish the model of PVDF sensor and analyze the relationship between output charge of PVDF and the force acting on it. Then to design the corresponding signal processing circuit, then simulate the circuit. Simulation results show design of the signal processing circuit is effective and modeling of PVDF sensor is correct. Thus, the paper provides a feasible solution in micro-force sensing and feedback control for micro-assembly and micro-manipulation.

\section{Acknowledgement}

This work is supported by National Nature Science Foundation of China (Grant No. 61203329). 


\section{References}

[1] Fatikow S., Seyfried J., Fahlbusch ST. Buerkle A. and Schmoeckel F., A flexible microrobot-based microassembly station[J]. Intelligent and Robotic, 2000, 27:135-169.

[2] Reid J. R., Bright V. M., Comtois J. H.Automated Assembly of Flip-up Micromirrors[R]. TRANSDUCERS'97 Chicage, 1997:347-350.

[3] Arne Sieber, Pietro Valdastri, and Keith Houston etc., A novel haptic platform for real time bilateral biomanipulation with a MEMS sensor for triaxial force feedback[J]. Sensors and Actuators, 2008, A142:19-27.

[4] B. B. Edin, L. Ascari, L. Beccai, and S. Roccella etc., Bio-inspired sensorization of a biomechatronicrobot hand for the grasp-and-lift task[J]. Brain Research Bulletin, 2008, 75:785-795.

[5] M. Ammi, and A. Ferreira, Biological cell injection visual and haptic interface[J]. Adv.Rob., 2006, 20(3): 283-304.

[6] Gab-Soon Kim, Development of a three-axis gripper force sensor and theintelligent gripper using it[J]. Sensors and Actuators, 2007, A137: 213-222.

[7] Ricardo P'erez, Nicolas Chaillet, and Krzysztof Domanski, Fabrication, modeling and integration of a silicon technology force sensor in a piezoelectricmicro-manipulator [J]. Sensors and Actuators, 2006, A128: 367-375.

[8] Zhao Yunhai, Characteristics and applications of PVDF piezoelectric film[J]. Sensor world, 2000, 3:23-26.

[9] Tang Luxin, Sensing and detection technology[M]. Beijing: Science Press, 2006.

[10]GANDELli A, OTTOBONI R. Charge Amplifiers for Piezoelectric Sensors[C]. IEEE, 1993, 11:18-20.

[11] Nie Dian, Li Beiyan, Nie Mengcheng, Su Xiaopeng, Multisim 12 simulation and design[M]. Beijing: Electronic Industry Press, 2014.

[12] Yang Suxing, Analog electronic technology brief tutorial (Third Edition)[M]. Beijing: Higher Education Press, 2006.Reference to a book: 